Discrete Comput Geom 37:205-212 (2007)

DOI: $10.1007 / \mathrm{s} 00454-006-1303-6$

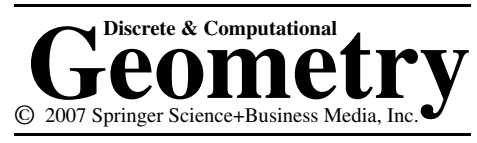

\title{
Optimal Arrangements in Packing Congruent Balls in a Spherical Container
}

\author{
Wlodzimierz Kuperberg \\ Department of Mathematics, Auburn University, \\ Auburn, AL 36849-5310, USA \\ kuperwl@auburn.edu
}

\begin{abstract}
What is the minimum radius $\varrho_{d}(n)$ of a spherical container in $\mathbb{R}^{d}(d \geq 2)$ that can hold $n$ unit balls, and how must the balls be arranged in such a container? This question is equivalent to: How should $n$ points be selected in the unit ball in $\mathbb{R}^{d}$ so that the minimum distance between any two of them be as large as possible, and what is that distance? Davenport and Hajós [DH], and, independently, Rankin [R], proved that if $F$ is a set of $d+2$ points in the unit ball in $\mathbb{R}^{d}$, then two of the points in $F$ are at a distance of at most $\sqrt{2}$ from each other. Rankin proved also that if $F$ consists of $2 d$ points in the ball such that the distance between any two of them is at least $\sqrt{2}$, then their configuration is unique up to an isometry, namely the points must be the vertices of a regular $d$-dimensional crosspolytope inscribed in the ball. However, if $d+2 \leq n \leq 2 d-1$, then the optimal arrangements of $n$ points (i.e., those that maximize the smallest distance between them) are not unique. Here we generalize the results from $[\mathrm{DH}]$ and $[\mathrm{R}]$ by describing all possible optimal configurations, unique or not, of $n=d+2, d+3, \ldots, 2 d$ points.
\end{abstract}

\section{Introduction}

We consider here the following Packing Problem: Given a positive integer $n$, find the minimum radius $\varrho_{d}(n)$ of a spherical container in $\mathbb{R}^{d}(d \geq 2)$ that can hold $n$ unit balls. Also, determine all possible configurations of the set of the centers of the $n$ balls (up to an isometry).

Observe that the centers of the $n$ unit balls packed in a spherical container of radius $\varrho$ lie in a ball of radius $\varrho-1$, and the distance between any two of the centers is at least 2 . Conversely, if $n$ points can be selected in a ball of radius $\varrho$ so that the distance between any two of the centers is at least 2, then the $n$ unit balls centered at these points form a packing in a spherical container of radius $\varrho+1$. Therefore for $n \geq 2$, our Packing Problem is equivalent to the Dispersion Problem: Find the maximum dispersion $\delta=\delta_{d}(n)$ over 
all $n$-point subsets of the unit ball in $\mathbb{R}^{d}$ (the unit $d$-ball, for short), and determine all optimal configurations corresponding to it, that is, all $n$-point subsets (up to isometry) of the ball whose dispersion is $\delta_{d}(n)$. Here by the dispersion (not to be confused with other notions by the same name found in statistics and other areas of mathematics) of a finite set $F$ containing at least two points we mean the minimum distance between any two of the points in $F$. The relationship between $\varrho=\varrho_{d}(n)$ and $\delta=\delta_{d}(n)$ is given by either of the equalities $\varrho=(\delta+2) / \delta$ and $\delta=2 /(\varrho-1)$. Henceforth we shall be concerned with the Dispersion Problem only.

The regular $d$-dimensional crosspolytope (the $d$-crosspolytope, for short) is the polytope dual to the $d$-dimensional cube. More directly, the $d$-crosspolytope can be described as the convex hull of the union of $d$ mutually perpendicular line segments of equal length, intersecting at their common midpoint. Obviously, the $d$-crosspolytope generated by $d$ mutually perpendicular segments of length 2 is inscribed in the unit sphere, and the edge-length of the crosspolytope is $\sqrt{2}$. Each of the $2^{d}$ facets of the crosspolytope is a regular $(d-1)$-dimensional simplex of edge-length $\sqrt{2}$. Since the only distances between vertices of the crosspolytope inscribed in the unit sphere are $\sqrt{2}$ and 2 , the dispersion of the set of its $2 d$ vertices is $\sqrt{2}$.

The following two theorems provide solutions to some cases of the Dispersion Problem:

Theorem $1[\mathrm{DH}],[\mathrm{R}]$. If $d+2$ points lie in the unit $d$-ball, then at least one of the distances between the points is smaller than or equal to $\sqrt{2}$.

Theorem $2[\mathrm{R}] . \quad$ Each optimal 2d-point subset of the unit d-ball is the set of vertices of a regular d-crosspolytope inscribed in the ball.

The two above theorems can be expressed as follows:

$$
\delta_{d}(d+2)=\delta_{d}(d+3)=\cdots=\delta_{d}(2 d)=\sqrt{2},
$$

and the optimal configuration of $2 d$ points is unique, forming the set of vertices of a $d$-crosspolytope.

Remark 1. The set of vertices of the $d$-crosspolytope inscribed in the unit $d$-ball is saturated, meaning that every point of the ball is at a distance smaller than $\sqrt{2}$ from at least one of the vertices. Therefore Theorem 2 implies that $\delta_{d}(2 d+1)<\sqrt{2}$.

Remark 2. Although in $[\mathrm{DH}]$ and $[\mathrm{R}]$ it is assumed that the points lie on the surface of the ball, this restriction is redundant (see the first part of the proof of Theorem 3 below). Before Rankin, Schütte and van der Waerden [SW] solved the three-dimensional case, also with the assumption that the points lie on the ball's surface.

Remark 3. The Dispersion Problem for $n \leq d+1$ in the unit $d$-ball has been solved by Rankin [R] as well: the points must be the vertices of the largest regular $(n-1)$ dimensional simplex inscribed in the ball. Thus, the optimal configuration is unique 
and

$$
\delta_{d}(n)=\sqrt{\frac{2 n+2}{n}} \quad \text { for } \quad n \leq d+1 .
$$

In this way, Rankin characterizes both the regular simplex and the regular crosspolytope, each by the optimality property of its set of vertices. Such characterizations fit well with Fejes Tóth's idea of genetics of regular figures, described in Part 2 of [FT] and supported by a collection of examples illustrating how "... regular arrangements are generated from unarranged chaotic sets by ordering effect of an economy principle, in the widest sense of the word" [FT, Preface, p. x].

The main result of this article is the following theorem about the structure of optimal configurations, in effect describing all of the nonunique ones for each of the cases $n=d+2, d+3, \ldots, 2 d-1$, as well as the unique one for the case $n=2 d$.

Theorem 3 (Main Theorem). Let $d$ and $k$ be integers such that $2 \leq k \leq d$. If $F$ is an optimal $(d+k)$-point subset of the unit $d$-ball, then: (1) every point of $F$ lies on the boundary of the ball, and (2) $\mathbb{R}^{d}$ can be split into the orthogonal product $\prod_{i=1}^{k} L_{i}$ of $k$ nondegenerate linear subspaces so that $L_{i}$ contains exactly $1+\operatorname{dim} L_{i}$ points from $F$ $(i=1,2, \ldots, k)$.

Here the product's being orthogonal means that $x y=0$ for every $x \in L_{i}, y \in L_{j}$ with $i \neq j$.

Remark 4. Theorem 3 is a common generalization of Theorems 1 and 2 .

Proof. Let $F$ be an optimal $(d+2)$-point subset of the unit $d$-ball. From Theorem 3, for $k=2$ we get $\mathbb{R}^{d}=L_{1} \times L_{2}$, where each of $L_{1}$ and $L_{2}$ contains a point from $F$, say $x$ and $y$, respectively, lying on the boundary of the ball. Since $L_{1}$ and $L_{2}$ are orthogonal, while $\|x\|=\|y\|=1$, we get $\|x-y\|=\sqrt{2}$, as needed for Theorem 1 .

Next, assume $k=d$. Then $\mathbb{R}^{d}=\prod_{i=1}^{d} L_{i}$, thus $\operatorname{dim} L_{i}=1$ for each $i=1,2, \ldots, d$, hence each $L_{i}$ intersects $F$ in a pair of antipodes of the ball. Then the mutual orthogonality of the lines $L_{i}$ implies that $F$ is the set of vertices of a regular $d$-crosspolytope inscribed in the ball.

Remark 5. Here is how Theorem 3 yields an explicit construction of all optimal configurations of $n=d+k$ points in the unit $d$-ball for $2 \leq k \leq d$. To obtain any one of them, first present $d$ as the sum of $k$ positive integers, $d=d_{1}+d_{2}+\cdots+d_{k}$. Then choose $k$ mutually orthogonal linear subspaces $L_{1}, L_{2}, \ldots, L_{k}$ so that $\operatorname{dim} L_{i}=d_{i}$ for $i=1,2, \ldots, k$. Finally, for every $i=1,2, \ldots, k$, place $d_{i}+1$ points in the intersection of $L_{i}$ with the boundary of the ball so that the shortest distance between the points is at least $\sqrt{2}$. If $d_{i}=1$, then there is only one choice for the two points "selected" in $L_{i}$ : they must be the antipodes at which $L_{i}$ intersects the ball's surface. However, if $d_{i} \geq 2$ for some $i$, then there are infinitely many choices for the corresponding $d_{i}+1$ points, generating infinitely many distinct configurations. Indeed, one can start with the 
"standard" placement of the $d_{i}+1$ points at the vertices of a regular $d_{i}$-simplex inscribed in the unit $d_{i}$-ball in $L_{i}$, and since the edge-length of the simplex is greater than $\sqrt{2}$, these points have some freedom to move on the surface of the ball.

\section{Notation and Preliminary Statements}

The cardinality of a set $A$ is denoted by card $A$. The origin $(0,0, \ldots, 0)$ of $\mathbb{R}^{d}$ is denoted by $o, \mathbf{B}^{d}$ denotes the unit ball, and $\mathbf{S}^{d-1}$ is the unit sphere, i.e., the boundary of $\mathbf{B}^{d}$ in $\mathbb{R}^{d}$. For any linear subspace $L$ of $\mathbb{R}^{d}$, the orthogonal complement of $L$ is the linear subspace $L^{\perp}=\left\{x \in \mathbb{R}^{d}: x y=0\right.$ for every $\left.y \in L\right\}$. The dimension of $L$ is denoted by $\operatorname{dim} L$. Obviously, $\operatorname{dim} L+\operatorname{dim} L^{\perp}=d$ for every linear subspace $L$ of $\mathbb{R}^{d}$.

Further, for any subset $M$ of $\mathbb{R}^{d}$, Conv $M$ denotes the convex hull of $M$. A subset $A$ of $\mathbb{R}^{d}$ is affine if $A$ contains every line determined by two points in $A$. Defined similarly to the convex hull, the affine hull of $M$ is denoted by Aff $M$. The interior of $M$ is denoted by Int $M$, and $\operatorname{Int}_{A} M$ denotes the interior of $M$ relative to Aff $M$.

For every $x \in \mathbf{S}^{d-1}$, the hyperplane normal to $x$ and containing $o$ is denoted by $H_{0}(x)$, and $H_{-}(x)$ denotes the half-space of $H_{0}(x)$ opposite to $x$. In other words,

$$
H_{0}(x)=\left\{y \in \mathbb{R}^{d}: x y=0\right\},
$$

and

$$
H_{-}(x)=\left\{y \in \mathbb{R}^{d}: x y \leq 0\right\} .
$$

Also, with each $x \in \mathbf{S}^{d-1}$ we associate the set $C(x)$ of points in $\mathbf{B}^{d}$ distant from $x$ by at least $\sqrt{2}$, that is,

$$
C(x)=\left\{y \in \mathbf{B}^{d}:\|x-y\| \geq \sqrt{2}\right\} .
$$

For $d=2$, the shape of $C(x)$ resembles a crescent, as seen in Fig. 1.

Next, we state a few propositions, simple enough to have their proofs omitted.

Proposition 1. $C(x) \subset H_{-}(x)$ for every $x \in \mathbf{S}^{d-1}$.

Proposition 2. $\quad C(x) \cap H_{0}(x)$ is a great $(d-2)$-sphere of $\mathbf{S}^{d-1}$. Specifically, $C(x) \cap$ $H_{0}(x)=H_{0}(x) \cap \mathbf{S}^{d-1}$.

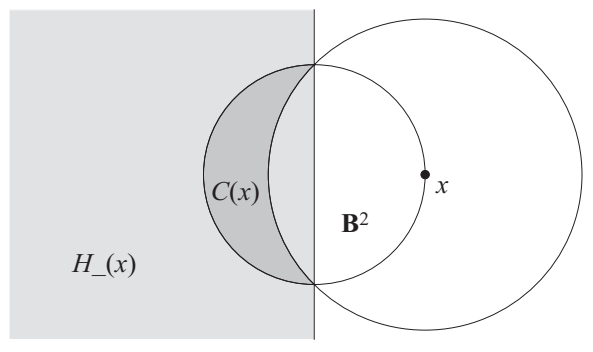

Fig. 1. The crescent $C(x)$. 
Proposition 3. If $S$ is a subset of $\mathbf{S}^{d-1}$ such that $o \in \operatorname{Int}(\operatorname{Conv} S)$, then

$$
\bigcap_{x \in S} H_{-}(x)=(\operatorname{Aff} S)^{\perp}=\{o\} .
$$

The above proposition is immediately generalized to:

Proposition 4. If $S$ is a subset of $\mathbf{S}^{d-1}$ such that $o \in \operatorname{Int}_{A}($ Conv $S)$, then

$$
\bigcap_{x \in S} H_{-}(x)=(\operatorname{Aff} S)^{\perp} .
$$

The above propositions imply directly the following intersection properties of the crescents $C(x)$ determined by points $x$ in a subset $S$ of $\mathbf{S}^{d-1}$.

Proposition 5. Suppose $S \subset \mathbf{S}^{d-1}$. If $o \in \operatorname{Int} \operatorname{Conv} S$, then $\bigcap_{x \in S} C(x)=\emptyset$.

Proposition 6. Suppose $S \subset \mathbf{S}^{d-1}$ and $s=\operatorname{dim}(\operatorname{Aff} S)$. If $o \in \operatorname{Int}_{A}(\operatorname{Conv} S)$, then $\bigcap_{x \in S} C(x)$ is the $(d-s-1)$-dimensional great sphere $\mathbf{S}^{d-1} \cap(\operatorname{Aff} S)^{\perp}$ of $\mathbf{S}^{d-1}$.

Suppose $A$ is a subset of $\mathbb{R}^{d}$ and $p$ is a point in the convex hull of $A$. Then, among all subsets of $A$ whose convex hull contains $o$, there exists a set $S$ (not necessarily unique) of minimum cardinality. By the well-known theorem of Carathéodory, the cardinality of such a minimal set $S$ is at most $d+1$.

Proposition 7. Suppose $S$ is a subset of $\mathbf{S}^{d-1}$ with o $\in$ Conv $S$, whose no proper subset's convex hull contains $o$. Then card $S=1+\operatorname{dim}(\operatorname{Aff} S)$ and $o \in \operatorname{Int}_{A}(\operatorname{Conv} S)$.

\section{The Proof}

Proof of Theorem 3. Assume that $2 \leq k \leq d$, and let $F=\left\{p_{1}, p_{2}, \ldots, p_{d+k}\right\}$ be an optimal subset of $\mathbf{B}^{d}$. As we noted before, the shortest distance between the $2 d$ vertices of the regular crosspolytope inscribed in $\mathbf{B}^{d}$ is $\sqrt{2}$. Therefore any two points in $F$ are at a distance of at least $\sqrt{2}$ apart.

We prove first that each point of $F$ lies on the boundary $\mathbf{S}^{d-1}$ of $\mathbf{B}^{d}$. Assume to the contrary that one of them, say $p_{1}$, does not. Since $F$ contains more than one point (in fact, $F$ contains at least four points), $p_{1} \neq o$. Let $p_{0}=p_{1} /\left\|p_{1}\right\|$. Observe that the set $C=\left\{y \in \mathbf{B}^{d}:\left\|y-p_{1}\right\| \geq \sqrt{2}\right\}$ is a subset of $C\left(p_{0}\right)$ and that $C \cap H_{0}\left(p_{0}\right)=\emptyset$. Therefore $F \cap \mathbf{S}^{d-1}$ is contained in an open hemisphere of $\mathbf{S}^{d-1}$, which allows $F$ to be shifted slightly, by a parallel translation in the direction of $p_{0}$, into the interior of $\mathbf{B}^{d}$, contradicting the optimality of $F$. This contradiction proves the first part of the theorem.

For a similar reason, $o \in \operatorname{Conv} F$; otherwise $F$ would be separated from $o$ by a hyperplane, hence it could be shifted into the interior of $\mathbf{B}^{d}$ by a parallel translation in the direction perpendicular to the hyperplane, towards $o$. 
The proof of the second part of the theorem proceeds by induction with respect to $d \geq 2$.

1. For $d=2$, we have $k=2$ and $\operatorname{card} F=4$. Let $S$ be a minimal subset of $F$ among such subsets whose convex hull contains $o$. Then $\operatorname{dim}(\operatorname{Aff} S$ ) is either 1 or 2 , thus $S$ consists of either two or three points. Since the latter case is impossible, as then we would have (Aff $S)^{\perp} \cap \mathbf{S}^{1}=\emptyset$, leaving no room for the fourth point of $F$, Aff $S$ is a line, and $S$ consists of two antipodes of $\mathbf{S}^{1}$. The remaining two points of $F$ lie on the line (Aff $S)^{\perp}$, thus $F$ is a regular 2-crosspolytope (a square) inscribed in $\mathbf{S}^{1}$.

2. For the inductive step we now assume that for some $d \geq 2$ our theorem holds in every dimension $d^{\prime} \leq d$ and for every $k=2,3, \ldots, d^{\prime}$. Let $F$ be an optimal set of $d+1+k$ points in $\mathbf{S}^{d}$, where $2 \leq k \leq d+1$. Just as in step 1 above, let $S$ be a minimum cardinality subset of $F$ among such subsets whose convex hull contains $o$. Let $L_{1}$ denote Aff $S$ and let $d_{1}=\operatorname{dim} L_{1}$. Then Conv $S$ is a $d_{1}$-dimensional simplex, $o \in \operatorname{Int}_{A}(\operatorname{Conv} S)$, $1 \leq d_{1} \leq d$, card $S=d_{1}+1$, and $F \backslash S \subset$ (Aff $\left.S\right)^{\perp}$. Therefore the set $F \backslash F^{\prime}$ consists of $d-d_{1}+k$ points lying on a unit sphere in a $\left(d-d_{1}+1\right)$-dimensional linear subspace of $\mathbb{R}^{d+1}$.

If $k=2$, then let $L_{2}=L_{1}^{\perp}$, and the proof is complete. In case $k>2$, the set $F \backslash S$, contained in $L_{1}^{\perp} \cap \mathbf{S}^{n}$, satisfies the assumptions of the theorem with $k^{\prime}=k-1$ and $d^{\prime}=d+1-d_{1} \leq d$. By the inductive assumption, $L_{1}^{\perp}$ can be split into an orthogonal product of $k-1$ subspaces $L_{2}, L_{3}, \ldots, L_{k}$ as stated in the conclusion of the theorem, and $\mathbb{R}^{d+1}$ splits into an orthogonal product of $k$ subspaces $L_{1}, L_{2}, \ldots, L_{k}$ as required.

Theorem 3 implies that if $n \leq 2 d$ is sufficiently close to $2 d$, then the optimal configuration of $n$ points in $\mathbf{B}^{d}$ must contain some mutually orthogonal antipodal pairs. This is expressed specifically in the following corollary, which in itself is a generalization of Theorem 2.

Corollary 1. Let $F$ be an optimal n-point subset of the unit d-ball. If $(3 d+s) / 2 \leq$ $n \leq 2 d$, then $F$ contains the set of vertices of a regular $s$-crosspolytope.

Proof. By Theorem $3, \mathbb{R}^{d}$ splits into an orthogonal product $\prod_{i=1}^{k} L_{i}$ of (nondegenerate) linear subspaces so that $L_{i}$ contains exactly $1+d_{i}$ points from $F$ for $i=1,2, \ldots, k$, where $d_{i}$ denotes $\operatorname{dim} L_{i}$ and $k=n-d$. Obviously, $\sum_{i=1}^{k} d_{i}=d$. Since $n \geq(3 d+s) / 2$, we get $2 k \geq d+s$, which implies that at least $s$ of the subspaces $L_{i}$ are of dimension 1. The corresponding $s$ antipodal pairs $L_{i} \cap F$ form the set of vertices of a regular $s$-crosspolytope.

\section{The Space of Optimal Configurations}

Theorem 3 implies that for $n=d+k$, where $2 \leq k \leq d-1$, the optimal sets have a number of degrees of freedom that decrease as $k$ increases and $d$ is fixed. For every $d$ and $n$, the space of optimal $n$-point sets $F$, furnished with the Hausdorff metric, is a compact space $\mathcal{F}_{d}(n)$ whose dimension is the number of degrees of freedom of points in a generic optimal set $F$. The quotient space of $\mathcal{F}_{d}(n)$ obtained by identifying optimal sets that are congruent is the space of optimal configurations, or the configuration space for short, denoted by $\mathcal{C}_{d}(n)$. 
We conjecture that $\mathcal{C}_{d}(n)$ is a (topological) polyhedron for every $d$ and $n$. For example, for every $d \geq 3$, the configuration space $\mathcal{C}_{d}(2 d-1)$ is a topological disk, being the space of congruence classes of nonobtuse triangles inscribed in the unit circle. The problem of describing explicitly the configuration space $\mathcal{C}_{d}(n)$ sounds interesting, but, in general, for $d \geq 4$ and $d+2 \leq n \leq 2 d-2$, it may be a difficult one.

The following theorem can be considered as a first step towards describing the topological nature of the configuration spaces. Recall that a topological space $X$ is pathwise connected if for every two points $x$ and $y$ in $X$, there is a continuous function $f:[0,1] \rightarrow X($ a path $)$ with $f(0)=x$ and $f(1)=y$.

Theorem 4. For every $d$ and $n \leq 2 d$, the configuration space $\mathcal{C}_{d}(n)$ is pathwise connected.

We precede the proof of this theorem with two lemmas.

Lemma 1. If $\Delta^{d}$ is a d-dimensional simplex inscribed in the sphere $\mathbf{S}^{d-1}$ and if every edge of $\Delta^{d}$ is of length at least $\sqrt{2}$, then $\Delta^{d}$ contains the sphere's center $o$.

Proof. For $d=1$ the lemma is obviously true. We proceed by induction and, for an indirect proof, assume that $d>1$ is the smallest dimension in which the lemma fails. Suppose then that a simplex $\Delta^{d}$ with vertices $v_{0}, v_{1}, \ldots, v_{d}$ satisfies the lemma's assumptions yet does not contain $o$. Then one of the $(d-1)$-dimensional faces of $\Delta^{d}$, say the face $\Delta_{0}=\left[v_{1}, \ldots, v_{d}\right]$, separates it from $o$. Let $H$ be the hyperplane determined by $\Delta_{0}$. Thus $\Delta_{0}$ lies in the $(d-2)$-dimensional sphere $S_{0}=H \cap \mathbf{S}^{d-1}$ and the radius of $S_{0}$ is smaller than 1 . Now, since every vertex of $\Delta_{0}$ is distant from $v_{0}$ by at least $\sqrt{2}$, we get $\Delta_{0} \subset H^{-}\left(v_{0}\right)$ (see Section 1, Proposition 1). Considering that $S_{0}$ separates $v_{0}$ from $o$, this implies that $\Delta_{0}$ is contained in an open hemisphere of $S_{0}$, hence does not contain the center $o^{\prime}$ of $S_{0}$. (The last statement is easily verified by reducing the situation to the two-dimensional case: just take the cross-section of all sets involved with the plane containing the points $o, o^{\prime}$, and $v_{0}$.) By expanding $S_{0}$ along with $\Delta_{0}$ by a homothety so that $S_{0}$ becomes a unit sphere, we obtain a contradiction with our inductive assumption.

Lemma 2. If $\Delta^{d}$ is a d-dimensional simplex inscribed in the sphere $\mathbf{S}^{d-1}$ and if every edge of $\Delta^{d}$ is of length at least $\sqrt{2}$, then $\Delta^{d}$ can be transformed continuously, by moving its vertices on the sphere and never allowing its edges to become shorter than $\sqrt{2}$, so that in the end the resulting simplex $\Delta^{\prime}$ has a pair of antipodal vertices.

Proof. For $d=1$ there is nothing to do, since the two vertices of a simplex $\Delta^{1}$ satisfying the assumptions already are each other's antipodes. Assume then, for an inductive proof, that the statement of the lemma is true for some $d \geq 1$, and thus let $\Delta^{d+1}$ be a $(d+1)$-dimensional simplex with vertices $v_{0}, v_{1}, \ldots, v_{d+1}$, inscribed in the sphere $\mathbf{S}^{d}$ and satisfying the edge-length assumption. According to Lemma $1, \Delta^{d+1}$ contains $o$. Let $\Delta_{0}^{d}$ be the face of $\Delta^{d+1}$ opposite to $v_{0}$, and let $H_{0}$ be the hyperplane containing $\Delta_{0}^{d}$. Let $w$ be the normal to $H_{0}$, pointing to the side of $H_{0}$ that contains $v_{0}$. In the first stage of transforming $\Delta^{d+1}$, keep $v_{0}$ fixed and rotate $H_{0}$ (with $\Delta_{0}^{d}$ in it) about 
$o$, so that the angle between $v_{0}$ and $w$ decreases monotonically until these two vectors become parallel. During the rotation, $\Delta_{0}^{d}$ moves rigidly, and no distance between $v_{0}$ and a vertex of $\Delta_{0}^{d}$ ever becomes smaller than $\sqrt{2}$. The next motion will shift the hyperplane in which the (rotated) $\Delta_{0}^{d}$ now lies by a parallel translation towards $v_{0}$ until that hyperplane reaches the center $o$ of $\mathbf{S}^{d}$. As before, $v_{0}$ remains stationary, but the distances from $v_{0}$ to the remaining vertices decrease until they all drop to $\sqrt{2}$. The resulting "new" simplex $\Delta^{d+1}$ has its face opposite to $v_{0}$ inscribed in the great sphere $\mathbf{S}^{d-1}$ of $\mathbf{S}^{d}$, to which $v_{0}$ is orthogonal. The last motion uses the inductive assumption: the face opposite to $v_{0}$ is transformed within the great sphere $\mathbf{S}^{d-1}$ so that a pair of antipodes is created among its vertices.

Proof of Theorem 4. For $n \leq d+1$ as well as for $n=2 d$, the configuration space $\mathcal{C}_{d}(n)$ consists of a single point. Assume therefore that $d+2 \leq n \leq 2 d-1$, and let $k=n-d$. Let $c^{*}$ denote the point in $\mathcal{C}_{d}(n)$ that is the configuration of $n$ points consisting of: either $n / 2$ (if $n$ is even) mutually perpendicular pairs of antipodes, or $(n-1) / 2$ mutually perpendicular pairs of antipodes plus one point perpendicular to each of the other $n-1$ points. In other words, $c^{*} \in \mathcal{C}_{d}(n)$ is either a complete set of vertices of a regular $(n / 2)$ dimensional crosspolytope, or the set of vertices of a regular $((n+1) / 2)$-dimensional crosspolytope with one vertex deleted.

We now show that for every $c \in \mathcal{C}_{d}(n)$ there is a path in $\mathcal{C}_{d}(n)$ connecting $c$ with $c^{*}$. Let $F$ be an optimal $n$-point subset of $\mathbf{S}^{d-1}$ representing the optimal configuration $c$ and let $F_{0}$ be the subset of $F$ consisting of all points in $F$ whose antipodes belong to $F$. Obviously, the antipodal pairs in $F_{0}$ are mutually orthogonal. If $F_{1}=F \backslash F_{0}$ contains at most one point, then $c=c^{*}$ and there is nothing to prove. Therefore we assume that $F_{1}$ contains at least two points. The set $F_{1}$ lies in (Aff $\left.F_{0}\right)^{\perp}$, a linear subspace of dimension $d_{1} \geq 1$. However, if $d_{1}$ were 1 , then $F_{1}$ would consist of a pair of antipodes, contradicting the definition of $F_{0}$. Thus $d_{1} \geq 2$, and $F_{1}$ contains at least $d_{1}+1$ points.

If card $F_{1}=d+1$, then Lemma 1 can be applied to transform $F_{1}$ continuously within the sphere $S_{1}=\left(\text { Aff } F_{0}\right)^{\perp} \cap \mathbf{S}^{d-1}$ so as to create another pair of antipodes in $F$, and if card $F_{1}>d+1$, then we can apply Theorem 3 to $F_{1}$ as a subset of $S_{1}$, and apply Lemma 1 to a subset $F_{2}$ of $F_{1}$ in one of the linear subspaces provided by Theorem 3 . Proceeding in this fashion until all, except at most one, points from the continuously transformed set $F$ have been grouped in pairs of antipodes, we will have constructed a path in $\mathcal{C}_{d}(n)$ connecting $c$ with $c^{*}$.

\section{References}

[AS] J. Aczél and T. Szele, Solutions to Problem 35 (in Hungarian), Mat. Lapok 3 (1952), 94-95.

[DH] H. Davenport and G. Hajós, Problem 35 (in Hungarian), Mat. Lapok 2 (1951), 68.

[FT] L. Fejes Tóth, Regular Figures, Pergamon Press, Oxford, 1964.

[R] R.A. Rankin, The closest packing of spherical caps in $n$ dimensions, Proc. Glasgow Math. Assoc. 2 (1955), 139-144.

[SW] K. Schütte and B.L. van der Waerden, Auf welcher Kugel haben 5, 6, 7, 8 oder 9 Punkte mit Mindestabstand 1 Platz?, Math. Ann 123 (1951), 139-144.

Received March 28, 2006, and in revised form June 22, 2006, and September 11, 2006.

Online publication February 9, 2007. 